\title{
Improved Mirror Coatings for use in the Lyman Ultraviolet to Enhance Astronomical Instrument Capabilities
}

\author{
Manuel A. Quijada ${ }^{a}$, Javier del Hoyo ${ }^{a}$, David R. Boris ${ }^{b}$, and Scott Walton ${ }^{b}$ \\ ${ }^{a}$ Optics Branch, Code 551, NASA Goddard Space Flight Center, \\ 8800 Greenbelt Rd., Greenbelt, MD 20771, USA; \\ ${ }^{b}$ Plasma Physics Division, Naval Research Laboratory, \\ 4555 Overlook Ave. SW, Washington, DC 20375, USA
}

\begin{abstract}
This paper will describe efforts at developing broadband mirror coatings with high performance that will extend from infrared wavelengths down to the Far-Ultraviolet (FUV) spectral region. These mirror coatings would be realized by passivating the surface of freshly made aluminum coatings with XeF2 gas in order to form a thin $\mathrm{AlF}_{3}$ overcoat that will protect the aluminum from oxidation and, hence, realize the high-reflectance of this material down to its intrinsic cut-off wavelength of $90 \mathrm{~nm}$. Improved reflective coatings for optics, particularly in the FUV region (90-120 nm), could yield dramatically more sensitive instruments and permit more instrument design freedom.
\end{abstract}

Keywords: LUVOIR, FUV, Absorption, Coatings, Spectral reflectance, $\mathrm{XeF}_{2}$, etching, fluorination

\section{INTRODUCTION}

Pure aluminum $(\mathrm{Al})$ is one of the few metals which has the highest intrinsic reflectance (when compared to other conventional metals such as $\mathrm{Au}$ or $\mathrm{Ag}$ ) over the broadest spectral range. For this reason, the recently commissioned Large Ultraviolet Optical Infrared (LUVOIR) observatory study that has been proposed as one option for the Astro2020 Decadal survey ${ }^{1}$ will undoubtedly require an Al-based coating on at least its primary mirror because of the broad spectral range coverage this mission has been envisioned $(90-5000 \mathrm{~nm})$. However, the naturally occurring aluminum oxide $\left(\mathrm{Al}_{2} \mathrm{O}_{3}\right)$ layer that forms on the surface of pure $\mathrm{Al}$ when exposed to air, severely limits its application as a reflecting coating in the far-ultraviolet (FUV) spectral region (90-200 $\mathrm{nm}$ ). The traditional solution to preserve the high reflectance of $\mathrm{Al}$ in the FUV is by protecting the non-oxidized surface with an overcoat of transparent material in this spectral region. Aluminum protected with fluorides such as $\mathrm{LiF}$ or $\mathrm{MgF}_{2}$ have been the most commonly used solutions. ${ }^{2,3}$ But below $102 \mathrm{~nm}$ down to $90 \mathrm{~nm}$, no transparent material is available to protect $\mathrm{Al}$ and coating mirror reflectance stays well below $30 \%$. But even above $102 \mathrm{~nm}$, the reflectance of protected $\mathrm{Al}$ is limited by the residual absorption of the fluoride overcoats and the hygroscopic nature of the LiF overcoat. Because of these shortcomings, the achievement of high-reflectance in broadband coatings, particularly in the FUV range, has been identified as an "Essential Goal" in the technology needs for the LUVOIR) surveyor observatory. Improved reflective coatings for optics in the FUV spectrum could yield dramatically more sensitive instruments and permit more instrument design freedom. ${ }^{4}$ This paper aims at reporting recent efforts to produce broadband mirror coatings with high performance that will extend from the infrared spectral region to FUV wavelengths. These mirror coatings would be realized by using a thin aluminum tri-fluoride $\left(\mathrm{AlF}_{3}\right)$ overcoat that will protect the aluminum from oxidation and, hence, realize the high-reflectance of this material down to its intrinsic cut-off wavelength of $90 \mathrm{~nm}$. We present the progress achieved to date and discuss the path forward to achieve high reflectance in the spectral region from 90 to $130 \mathrm{~nm}$ without degrading performance in the visible and NIR regions, taking into account durability concerns when the mirrors are exposed to normal laboratory conditions.

A number of experiments have been reported in the literature to improve the deposition of $\mathrm{MgF}_{2}$ and $\mathrm{LiF}$ films that are intended as a protective overcoat for $\mathrm{Al}$ mirrors and interference filters in the FUV) spectral

Send correspondence to M.A.Q.: E-mail: manuel.a.quijada@nasa.gov, Telephone: +1 3012863544 
range. ${ }^{5,6}$ To maximize reflection of either the $\mathrm{Al}+\mathrm{MgF}_{2}$ or $\mathrm{Al}+\mathrm{LiF}$ coating pairs, the key is to produce a thin film of $\mathrm{MgF}_{2}$ (or $\mathrm{LiF}$ ) that is minimally absorbing in the FUV with a thickness of approximately a quarter of the operating wavelength in the $90-180 \mathrm{~nm}$ range.

The importance of producing high-performance reflecting coatings in the 90 to $180 \mathrm{~nm}$ range is hard to be overstated, since research in the FUV is relevant to many aspects of NASA's Cosmic Origins program, particularly the Astrophysics Science Area Objective 2: "Understand the many phenomena and processes associated with galaxy, stellar, and planetary system formation and evolution from the earliest epochs to today." Many of the resonance lines for both low-ionization and high-ionization states of common atoms are found largely in this region. Some lines are found at wavelengths greater than $120 \mathrm{~nm}$ but often their interpretation requires transitions with different oscillator strengths or different ionization states that are found in the FUV. Furthermore, the electronic ground-state transitions of $\mathrm{H}_{2}$ are only found below $115 \mathrm{~nm}$. Hydrogen gas is the most abundant molecule in the universe and is the fundamental building block for star and planet formation. The absorption lines of deuterium (D) and the molecule HD are found only in the FUV region as well. Understanding the abundance of D is an important test of Big Band cosmology and of chemical evolution over cosmic time.

The region from 90 to $115 \mathrm{~nm}$ has only been explored by a handful of NASA astronomy missions - Copernicus (OAO-3) in the 1970s, Hopkins Ultraviolet Telescope (HUT) and the Orbiting Retrievable Far and Extreme Ultraviolet (ORFEUS) in the 1990s, and the Far Ultraviolet Spectroscopic Explorer (FUSE) in the 2000s. The FUSE observing mission was the most extensive by far, but it was limited by modest effective area $\left(20 \mathrm{~cm}^{2}\right.$ below $100 \mathrm{~nm}$ to $55 \mathrm{~cm}^{2}$ above $102 \mathrm{~nm}$ ) and a modest spectral resolution (R 20,000). Moreover, FUSE made significant strides in mapping variations in $\mathrm{D} / \mathrm{H}$ in the galaxy but lacked the sensitivity to study $\mathrm{D} / \mathrm{H}$ in the inter-galactic medium (IGM). This lack of sensitivity was due to low reflectance of the available coatings. The reflectivity of the $\mathrm{Al}+\mathrm{LiF}$ coatings was $50 \%$ at launch, while that of the $\mathrm{SiC}$ coatings was $30 \%$. Improved reflectivity in itself would bring enormous gains in throughput, and the benefits of more capable optical designs enabled by higher reflectivity would address the shortcomings noted above and thus bring further gains in sensitivity.

There have been recent advances in the production of $\mathrm{Al}$ coatings protected with metal fluoride dielectric films that reach reflectance over $90 \%$ at the Lyman-alpha wavelength $(\lambda=1216 \mathrm{~nm})$. The reason for these gains has been attributed to the deposition of more dense overcoat layers of either $\mathrm{MgF}_{2}$ or $\mathrm{LiF}$ with intrinsic cut-off wavelengths closer to the theoretical limit. ${ }^{6}$ The depositon method used to produce these samples is via a physical vapor deposition (PVD) process of both the $\mathrm{Al}$ and the metal fluoride layer, with the key difference that the latter metal-fluoride layer is done with the substrate at elevated temperature $\left(\approx 260{ }^{\circ} \mathrm{C}\right) .^{7}$

In this paper, we report on the efforts to develop a technique to passivate oxide-free aluminum (Al) samples (prepared via a PVD method) by etching them with xenon difluoride $\left(\mathrm{XeF}_{2}\right)$ gas. This approach is motivated by earlier reports ${ }^{8,9}$ that have demonstrated these fluorinations experiments will result in the formation of a thin layer of $\mathrm{AlF}_{3}$, which is a dielectric material with a low refractive index with excellent transparency in the FUV spectral region. The passivation of oxide-free $\mathrm{Al}$ films with $\mathrm{AlF}_{3}$ has the potential to realize the high reflectance of $\mathrm{Al}$ down to cut-off wavelength of the $\mathrm{AlF}_{3}$ compound. This paper also discuss the feasibility of using an electron beam (e-beam) generated plasma to etch and fluorinate the surface of already oxidized $\mathrm{Al}$ surfaces. The paper is organized as follows: Section 2 gives a description of the experimental techniques to achieve the fluorination of the $\mathrm{Al}$ coatings using the $\mathrm{XeF}_{2}$ gas (Sec. 2.1). Section 2.2 provides a description of the Physical Vapor Deposition (PVD) technique, while Sec. 2.3 gives details on instrumentation used for spectral testing including a description of a vacuum ultraviolet (VUV) spectrometer. Section 2.4 describes and alternative method for achieving the passivation of the $\mathrm{Al}$ surface by using an electron beam (e-beam) generated plasma developed at the US Naval Research Laboratory. ${ }^{10}$ Section 3 is devoted to present results of these efforts. We start in Sec. 3.1 by presenting results and discussion of the attempt to thin-out the dielectric layers of $\mathrm{Al}$ mirror coatings protected with $\mathrm{LiF}$ and $\mathrm{AlF}_{3}$ as well as removing the native oxide layer of bare $\mathrm{Al}$ samples. Section 3.2 is devoted to present reflectance results of a second bare $\mathrm{Al}$ sample that was exposed to $\mathrm{XeF}_{2}$ gas. Section 3.3 discusses the viability of using $\mathrm{AlF}_{3}$ as dielectric overcoat to provide long-term durability of $\mathrm{Al}$ reflectors. The paper ends with some concluding remarks and plans for future work. 


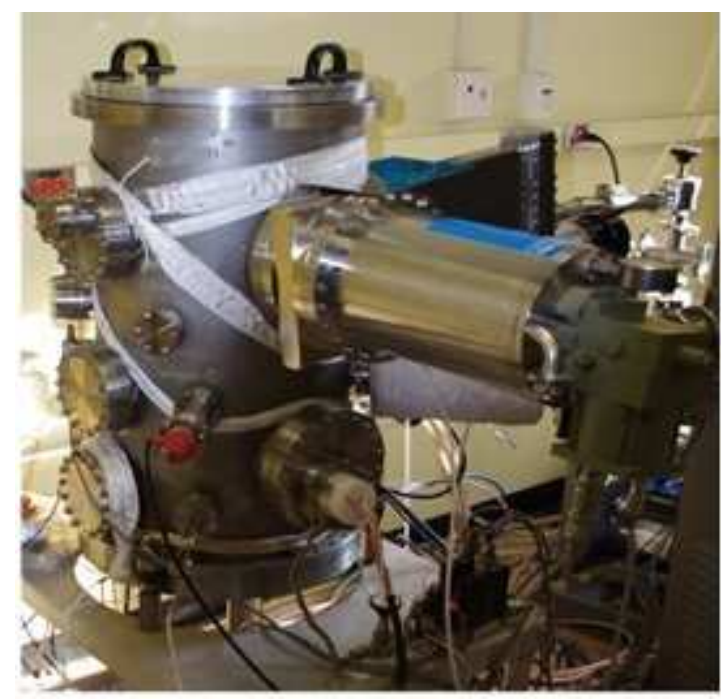

Figure 1. Image of the 0.5-meter vacuum chamber to be used for the Al deposition and fluorination experiments.

\section{EXPERIMENTAL TECHNIQUES}

This section is devoted to provide experimental details of the approach for using the etching techniques described earlier (namely $\mathrm{XeF}_{2}$ and electron beam plasma etching) to realize Al mirrors with high reflectance performance over the full spectral range and particularly in the FUV region.

\section{1 $\mathrm{XeF}_{2}$ Etching}

The $\mathrm{XeF}_{2}$ compound is widely used as an etching agent of sacrificial layers in the microelectromechanical (MEMs) technology. This material is known for its highly selective, isotropic etching. For example, Zhang et.al reported that $\mathrm{XeF}_{2}$ was used for the release of both $\mathrm{Al}$ and $\mathrm{Al}_{2} \mathrm{O}_{3}$ surfaces. ${ }^{11}$ In these experiments, the surfaces were first prepared by producing samples of these compounds with a cap layer of silicon ( $\mathrm{Si}$ ) (e.g. $\mathrm{Si} / \mathrm{Al}$ and $\mathrm{Si} / \mathrm{Al}_{2} \mathrm{O}_{3}$ ). The top sacrificial $\mathrm{Si}$ layer was removed by etching it away with $\mathrm{XeF}_{2}$ gas according to the formula:

$$
\mathrm{Si}+2 \mathrm{XeF}_{2}->2 \mathrm{Xe}+\mathrm{SiF}_{4}
$$

In this study, the chemical interaction between $\mathrm{XeF}_{2}$ and $\mathrm{Al}$ and $\mathrm{Al}_{2} \mathrm{O}_{3}$ surfaces was studied by in situ x-ray photoelectron spectroscopy (XPS). The surface adhesion and chemical composition of these samples were also measured after the exposure to air or annealing (at $200^{\circ} \mathrm{C}$ under vacuum) and the results revealed the presence of $\mathrm{AlF}_{3}$. Aluminum samples prepared this way have never been (to the best of our knowledge) tested in terms of their FUV reflectivity. Therefore, this paper reports on a program to conduct a series of fluorination experiments of oxide-free $\mathrm{Al}$ coatings with different $\mathrm{XeF}_{2}$ exposure to determine the optimum cycle of treatments that will produce a thin layer of $\mathrm{AlF}_{3}$ on top of the $\mathrm{Al}$ and to verify both chemical composition and reflectance performance.

\subsection{Physical Vapor Deposition}

The first part of this effort includes retrofitting an existing 0.5-meter high-vacuum chamber with the necessary hardware for producing high-quality Al films. Figure 1 shows an image of this chamber that is located in the Components Laboratory in the Optics Branch (Code 551) at the Goddard Space Flight Center. This chamber has been used in the past for performing Ion Beam Sputtering coatings and, as can been seen in this figure, is equipped with a cryo-pump, gate valve, and a number of ports and feed-through that make it highly configurable for various uses. The Al coating process in this chamber is based on a Physical Vapor Deposition (PVD) method where the $\mathrm{Al}$ is placed in a resistive bowl (made out of Tungsten) with electrical wires attached to it. A current is passed through this bowl until the $\mathrm{Al}$ melts and evaporates onto the substrate sample on top. One parameter that determines the quality of the deposited $\mathrm{Al}$ is a high vacuum (which is typically as maintained from the low 

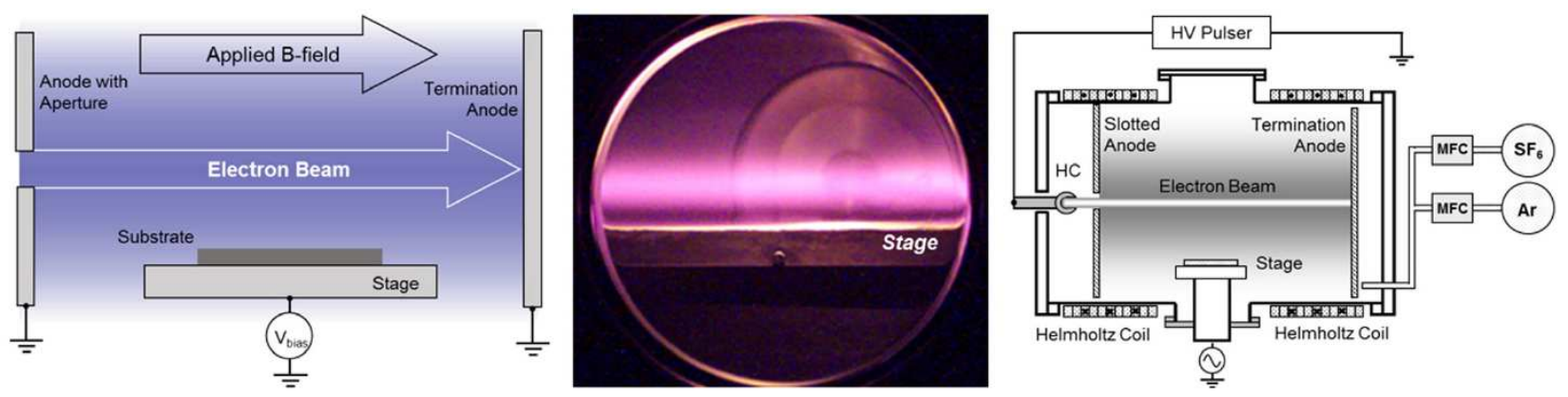

Figure 2. The US Naval Research Laboratory's Large Area Plasma Processing System (LAPPS), which employs an electron beamgenerated plasma. (Left) An illustration of the system, (Middle) an image of the plasma through a 6 inch port, and (Right) a schematic diagram of the processing reactor used in this work (after Reference [12]).

$10^{-7}$ Torr to the high $10^{-8}$ Torr range) during the coating process. We also ensured this vacuum chamber would have very low levels of residual water vapor and oxygen in order to minimize the possibility of oxidation of the $\mathrm{Al}$ layer. The $\mathrm{Al}$ deposition rate was maintained at $100 \AA$ per second or higher, in order to obtain a denser $\mathrm{Al}$ film.

A second element in this effort is to have a safe and controllable delivery system for the $\mathrm{XeF}_{2}$ gas inside the chamber. In this task, the $\mathrm{XeF}_{2}$ canister is attached to an expansion vessel with a solenoid control pneumatic valve for pulsed or continued delivery of the gas. The expansion chamber is connected to one of the 4.25 inch ports on the side of the chamber. Various experiments will be conducted to determine the optimum dosage of $\mathrm{XeF}_{2}$ gas for an $\mathrm{Al}$ coating that would have the best reflectance performance in the FUV. Once these exposures have been completed, $\mathrm{N}_{2}$ gas will be used to purge out the excess fluorine containing gases from inside of the chamber. In addition, and abatement system is used in the exhaust port of a corrosion resistant mechanical pump so that not fluorine containing gas would be discharged into the environment.

\subsection{FUV Reflectance Measurements}

The instrument used to measure reflectance as a function of wavelength is a MacPherson Vacuum Ultraviolet (VUV) 225 spectrophotometer. This spectrometer is a one-meter high-vacuum monochromator designed to provide access to the spectral range from $30 \mathrm{~nm}$ to $325 \mathrm{~nm}$ with a 1200 Grooves/mm grating. Effective coverage of the spectral range is dependent upon factors such as the optical coatings, grating efficiency, order-sorting filters, light source and detector. The spectrometer is equipped with a windowless hydrogen-purged light source, which provides discrete $\mathrm{H}_{2}$ emission lines between $90 \mathrm{~nm}$ and $160 \mathrm{~nm}$ and a continuum above these wavelengths. The detector, which is housed inside a sample-holder compartment consists of a photomultiplier cathode tube (PMT) connected to a light-pipe for feeding the light signal coming out of the monochromator. The light pipe has a fluorescence and high quantum efficiency coating of sodium salicylate that is used to convert the FUV radiation into visible light. The maximum emission efficiency of this coating matches that of the PMT sensitivity curve that is close to $420 \mathrm{~nm}$.

The reflectance measurement is performed by first collecting a $100 \%$ reference measurement by moving the detector in front so that it captures all the energy coming from the optical path inside the spectrometer. The sample to be measured is then inserted in the optical path of the beam and the detector is positioned to measured the energy reflected off the surface of the sample (at a near-normal angle of incidence close to $10^{\circ}$ ). The final step is to take the numerical ratio of the signal reflected off the sample to the $100 \%$ reference signal collected above. The resulting ratio provides the absolute reflectance response from the sample without the need of a reference standard.

\section{4 e-Beam Plasma Etching}

A second etching process to consider for removing the native oxide layer and fluorinate the surface of $\mathrm{Al}$ samples is based on the large area plasma processing system (LAPPS) which has been developed at the Naval Research 


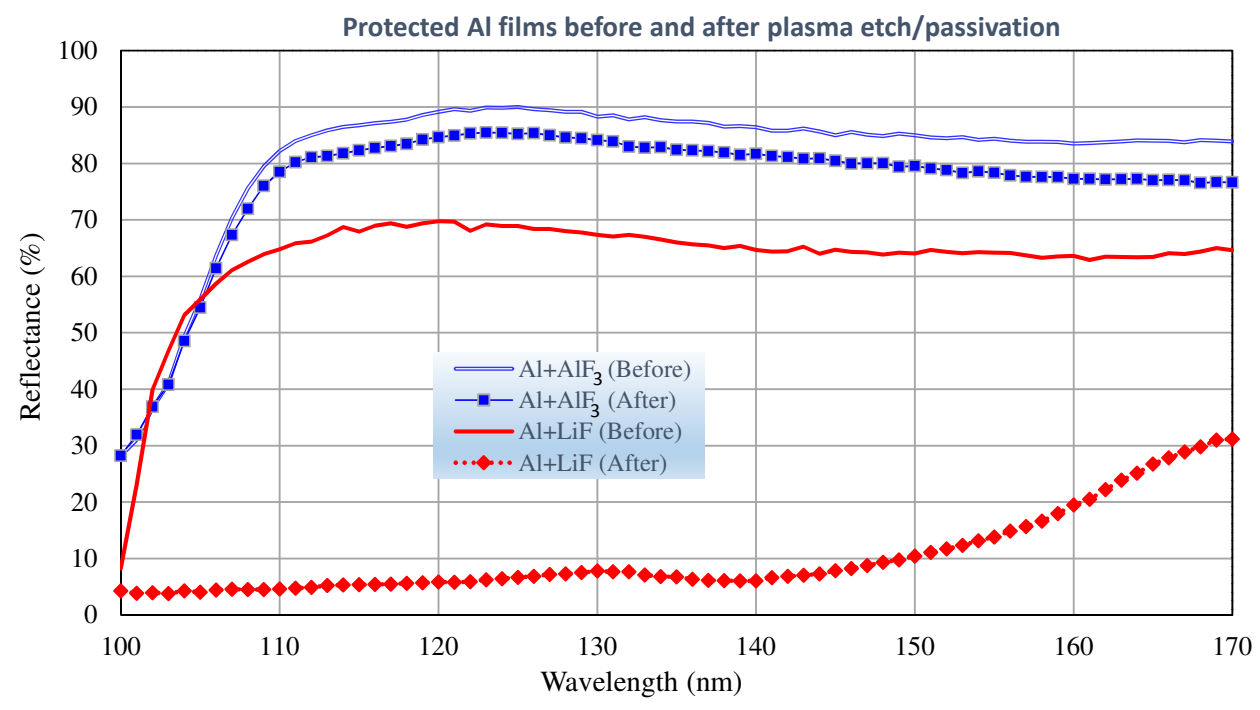

Figure 3. FUV reflectance of $\mathrm{AL}+\mathrm{AlF}_{3}$ and $\mathrm{Al}+\mathrm{LiF}$ smaples before and after etching with the LAPPS e-beam plasma system at NRL.

Laboratory (NRL). The LAPPS system employs magnetically-collimated, sheet-like e-beams to generate similarly sized plasma sheets for materials processing. ${ }^{12,13}$ A schematic of the processing system used in this work is shown in Fig. 2. The base configuration is relatively simple, consisting of an e-beam source, slotted anode, termination anode, sample holder, and magnetic field coils. Typically, the e-beams are $1-3 \mathrm{kV}$ with current densities of 1-5 $\mathrm{mA} / \mathrm{cm}^{2}$. Co-axial magnetic fields of 100-300 Gauss are used to collimate the e-beam and thus improve uniformity along its length. ${ }^{14}$ These parameters are sufficient to produce uniform plasma sheets compatible with large-scale systems $\left(\approx 1 \mathrm{~m}^{2}\right)$ operating at low pressures $(<100 \mathrm{mTorr})$.

The plasma processing system and its operation for the modification of materials has been previously described in detail elsewhere. ${ }^{10}$ Briefly, the system vacuum was maintained by a $250 \mathrm{~L} / \mathrm{s}$ turbo pump, with a base pressure $\approx 10^{-6}$ Torr. The background pressure was achieved by introducing $\mathrm{Ar}$ (purity $>99.9999 \%$ ) and $\mathrm{SF}_{6}$ (purity $>99.9999 \%$ ) through mass flow controllers and throttling the pumping speed with a variable conductance iris. The e-beam was produced by applying a $-2.5 \mathrm{kV}$ voltage to a linear hollow cathode for a given duration. The emergent beam passes through a slot in a grounded anode, traverses the gas, and it is then terminated at a second grounded anode located further downstream. The e-beam volume between the two anodes defines the ionization source volume, with the dimensions set by the slot size $\left(1 \times 7 \mathrm{~cm}^{2}\right)$ and the anode-to-anode length (40 $\mathrm{cm})$. A magnetic field of 150 Gauss was produced by a set of external coils.

The samples were placed on a processing stage located $3.0 \mathrm{~cm}$ from the e-beam axis. The stage was grounded and held at room temperature. The plasma potential within the processing volume was controlled by positively biasing the termination anode. This allows for precise control of the kinetic energy of incident ions on the substrate. ${ }^{10}$ Since the surface of the $\mathrm{Al}$ thin-film samples is conductive, RF biasing is not required as long as electrical contact between the substrate and processing stage is maintained. This work describes the salient features of these plasmas produced in mixtures of argon and sulfur hexafluoride $\left(\mathrm{SF}_{6}\right)$ and their use in silicon nitride etching, with particular attention paid to developing processing parameters relevant to atomic layer processing.

\section{RESULTS}

\subsection{Electron-Beam Plasma Etching}

Three Al thin films samples were treated with the LAPPS process described in Sec. 2.4 at NRL. The characteristics of the first sample is $\mathrm{Al}$ film $(\approx 50 \mathrm{~nm})$ protected with a LiF layer $(\approx 22 \mathrm{~nm})$. The second is an $\mathrm{Al}$ coating protected with $\mathrm{AlF}_{3}\left(\mathrm{Al}: \approx 50 \mathrm{~nm} ; \mathrm{AlF}_{3}: \approx 24.5 \mathrm{~nm}\right)$. The third sample is a bare Al layer $(\approx 70 \mathrm{~nm})$ 


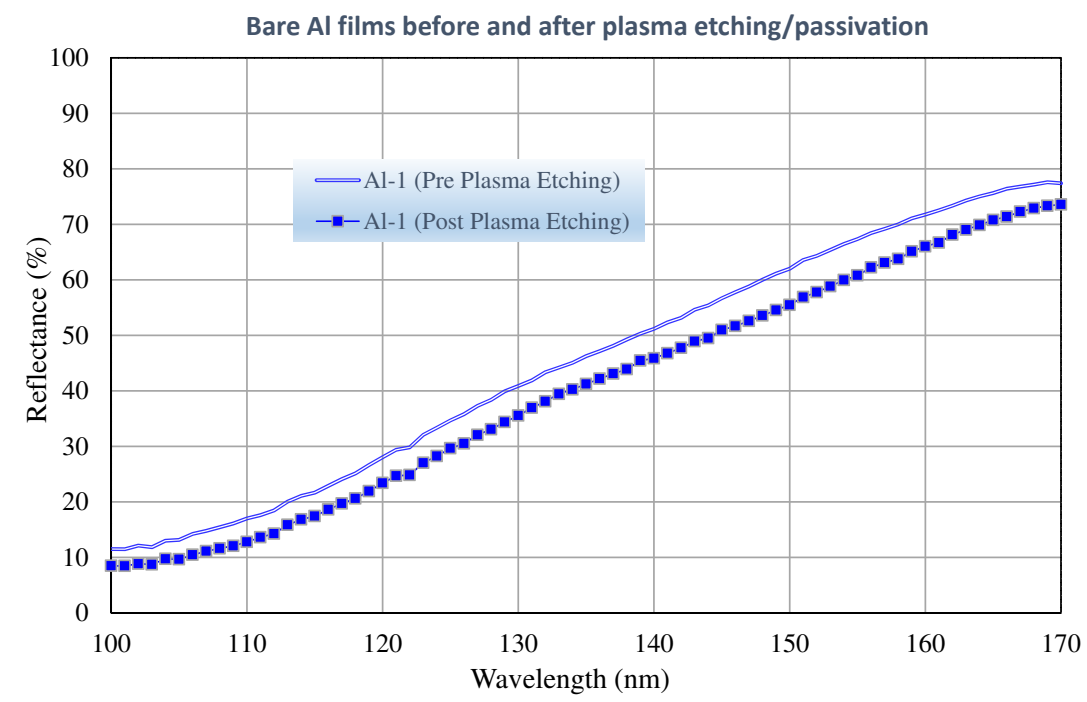

Figure 4. FUV reflectance of bare Al sample before and after treatment with the LAPPS e-beam plasma system at NRL.

with an estimated native $\mathrm{Al}_{2} \mathrm{O}_{3}$ layer that is around 2-4 nm thick. The native oxide sample was first exposed to an argon (Ar) plasma with a termination anode bias of $+30 \mathrm{~V}$ for 60 seconds. This was then followed by an exposure to a 20:1 mixture of argon (Ar) and sulfur hexafluoride $\left(\mathrm{SF}_{6}\right)$ with no termination anode bias for 20 seconds. The aluminum thin film samples with the $\mathrm{AlF}_{3}$ and $\mathrm{LiF}$ protection layers were exposed to an Ar plasma with a termination anode bias of $+20 \mathrm{~V}$ for 30 seconds. The neutral pressure, measured with a capacitance monometer, during processing was 75 mTorr, in the pure Ar case, whereas with the 20:1 mixture of Ar:SF 6 the pressure was 81 mTorr.

Figure 3 displays the FUV reflectance before and after two of the protected samples with $\mathrm{LiF}$ and $\mathrm{AlF}_{3}$ were treated with the LAPPS process. The results in this figure show a significant suppression in reflectance for both of these samples. The sample coated with the LiF layer suffered the most severe degradation, where the reflectance went from an average value (in the 100-170 $\mathrm{nm}$ range) from $62 \%$ (before e-beam plasma treatment) to $10 \%$ afterwards. The reduction in the sample protected with $\mathrm{AlF}_{3}$ was less severe in going from $81 \%$ (before) to $76 \%$ (after). The intended purpose in treating both of these samples with the LAPPS process was to thinout the top layer (at an atomic level) with the goal of boosting reflectance close to the cut-off wavelengths of the respective $\mathrm{LiF}$ and $\mathrm{AlF}_{3}$ compounds. However, the treatment in both samples caused an across-the-board reduction in reflectance and this may suggest significant changes in the chemical composition of the top-most layers. Chemical analyses on both samples are planned in the future in order to determine changes in the chemical compositions of the nominal $\mathrm{LiF}$ and $\mathrm{AlF}_{3}$ layers.

Figure 4 shows the results of etching the bare Al films that had the native oxide layer by following the prescription described above. These results also show a 5\% reduction in the average FUV reflectance: $45 \%$ (before) vs. $40 \%$ (after). Again, it appears that the intended purpose of removing the native oxide layer and passivating it with $\mathrm{AlF}_{3}$ was not successful. Further research will be pursued in order to determine the optimum parameters as these initial experiments were quite conservative (in terms of low ion energies) for achieving the intended outcome of removing the native oxide layer and capping the surface with an $\mathrm{AlF}_{3}$ overcoat.

\subsubsection{Reasons for choosing these process conditions:}

The choice of the process conditions described in Sec. 3.1 were driven by the competing needs to both remove the native oxide layer from the surface of the $\mathrm{Al}$ thin film while also leaving the reflective qualities of the thin film either unchanged or enhanced. Thus low ion bombardment energies were chosen for the Ar clean step to ensure minimal roughening of the $\mathrm{Al}$ thin film, with the hope that the $30 \mathrm{eV}$ ions would be sufficient to remove the $2-4 \mathrm{~nm}$ thick native oxide layer from the bare $\mathrm{Al}$ sample. The subsequent exposure to the $\mathrm{Ar} / \mathrm{SF}_{6}$ mixture 


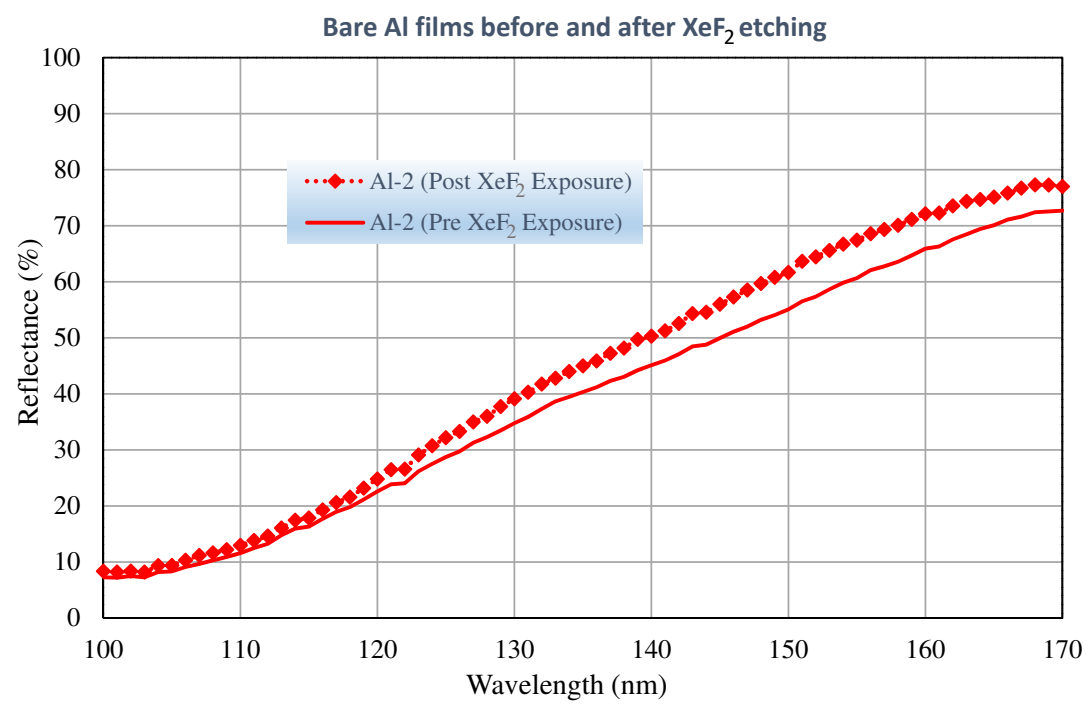

Figure 5. FUV reflectance of bare $\mathrm{Al}$ sample before and after exposure with $\mathrm{XeF}_{2}$ gas.

was intended to provide a very low energy flux of $\mathrm{F}_{2}^{+}$, and $\mathrm{SF}_{X}^{+}$ions combined with exposure to $\mathrm{F}$, and $\mathrm{F}_{2}$ radicals to generate an $\mathrm{AlF}_{3}$ surface passivation layer. ${ }^{15}$

These initial experiments of exposing an existing $\mathrm{Al}$ film coated with $\mathrm{AlF}_{3}$ thin film layer to the Ar plasma was meant to demonstrate the ability to control the thickness of these layers through post-deposition low damage etching. Further experimentation will be performed to determine the optimum deposition that would adjust the overlaying $\mathrm{AlF}_{3}$ thickness without causing damages to the surface.

\section{2 $\mathrm{XeF}_{2}$ Etching of Bare Al}

Figure 5 shows the results of treating a second $\mathrm{Al}$ (bare) sample in a $\mathrm{XeF}_{2}$ reactor located in the Detector Branch (Code 553) at GSFC. The treatment consisted of exposing the sample with a total of 50 cycles (10 seconds per cycle) with a $\mathrm{XeF}_{2}: \mathrm{N}_{2}$ mixture with a $1: 5$ ratio. These results indicate an increase in the average FUV reflectance that went from $39 \%$ (pre-exposure) to $43 \%$ (post-exposure). These results (although modest) points to a measurable change in the composition of the native oxide layer that may include the formation of $\mathrm{AlO}_{x} \mathrm{~F}_{y}$ ions on the surface as reported by Roodenko et.al. where a mixture of $\mathrm{Al}_{2} \mathrm{O}_{3}$ and $\mathrm{AlO}_{x} \mathrm{~F}_{y}$ ion species were found on the surface of samples treated with $\mathrm{XeF}_{2}$ gas by infrared spectroscopy through the detection of infrared-active modes associated with these compounds. ${ }^{9}$

\section{3 $\mathrm{AlF}_{3}$ as a Protection Layer}

This section discusses the viability of using $\mathrm{AlF}_{3}$ as a protection layer to realize high $\mathrm{FUV}$ reflectance for $\mathrm{Al}$ mirrors. As mentioned before, the $\mathrm{AlF}_{3}$ compound is a dielectric material with a low refractive index and wide band gap $>10 \mathrm{eV}$. It also has high transmission at infrared (IR), ultraviolet (UV) and deep UV wavelengths. These properties make this compound an attractive option to protect $\mathrm{Al}$ coatings in order to provide a broadband reflector. Because it has a slightly larger band gap (in comparison to $\mathrm{MgF}_{2}$ ), the $\mathrm{AlF}_{3}$ will give access to lower wavelengths when used as a protection layer on FUV reflectors. In addition, $\mathrm{AlF}_{3}$ is less hygroscopic than $\mathrm{LiF}$, so it will be a more environmentally stable coating. Finally, the lowest surface roughness, even at high deposition rates, makes $\mathrm{AlF}_{3}$ well suited for use in protected and enhanced FUV Al mirrors. ${ }^{16}$ Because of these potential advantages, we performed coating runs using $\mathrm{AlF}_{3}$ as a protection layer for $\mathrm{Al}$ coatings.

The left side of Fig. 6 shows an $\mathrm{Al}+\mathrm{AlF}_{3}$ sample prepared with the 3 -step PVD method described in reference [7]. The coating parameters for this sample are as follows: an Al layer that is $70 \mathrm{~nm}$ thick, while the $\mathrm{AlF}_{3}$ overcoat is roughly $24 \mathrm{~nm}$. The average FUV reflectance in the range shown in this figure is around $74 \%$, while reaching a peak reflectance close to $90 \%$ at the Lyman-Alpha wavelength $(121.6 \mathrm{~nm})$. Furthermore, the reflectance remained 

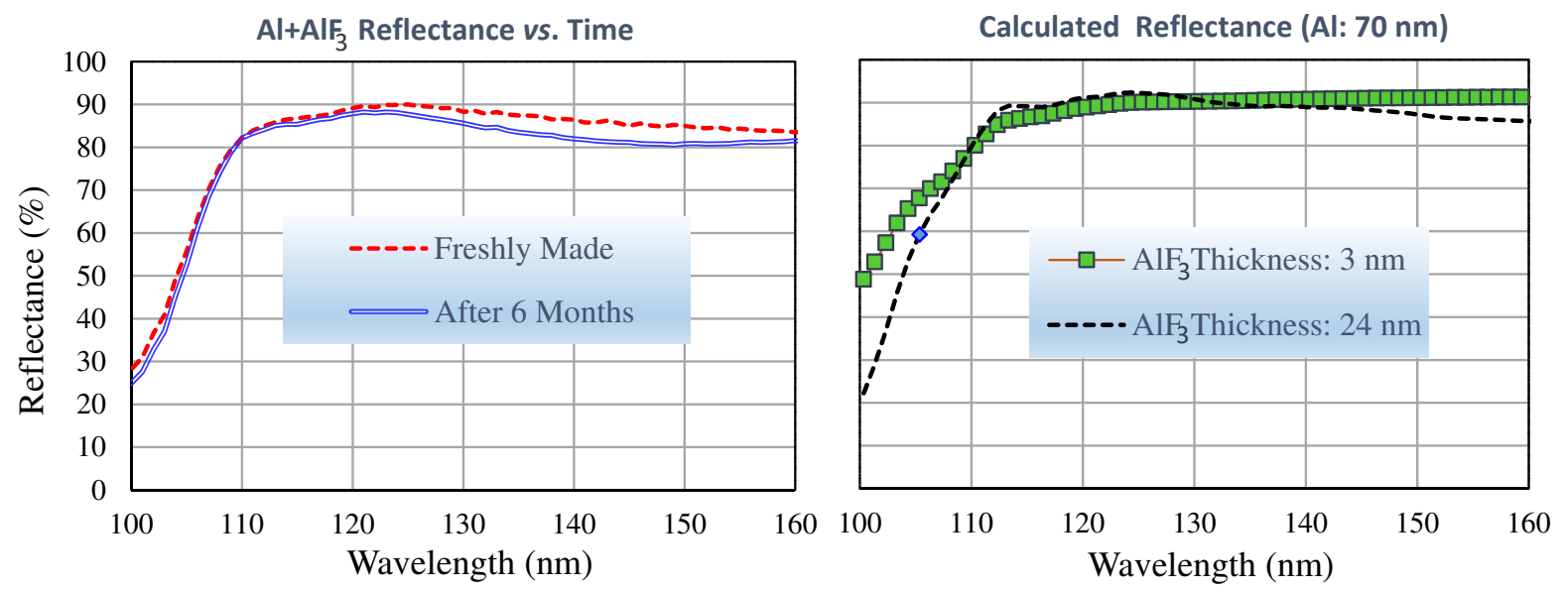

Figure 6. (Left) The FUV reflectance of a $\mathrm{Al}+\mathrm{AlF}_{3}$ sample that was prepared with the conventional PVD process. These results show vey little changes after sample was kept in ambient laboratory conditions for more than six months. (Right) Predicted reflectance based on thickness parameters (Al: $70 \mathrm{~nm} ; \mathrm{AlF}_{3}: 24 \mathrm{~nm}$ ) of sample shown on the left. The second (green squares) curve shown on the righ panel illustrate the predicted performance of a sample with an $\mathrm{AlF}_{3}$ layer $3 \mathrm{~nm}$ thick.

virtually unchanged after keeping the sample in ambient laboratory conditions (40-50\% relative humidity) for more than 6 months (blue curve in Fig. 6). These results would certainly suggest that $\mathrm{AlF}_{3}$ could have the potential to replace either $\mathrm{MgF}_{2}$ or $\mathrm{LiF}$ as the material of choice for protecting $\mathrm{Al}$ for applications in the FUV spectral range. In addition, the right side panel of Fig. 6 illustrates good agreement in the calculated reflectance for an $\mathrm{Al}+\mathrm{AlF}_{3}$ sample with thin-film parameters as the data shown in the left side of this figure. Finally, the green square in Fig. 6 (right side) shows the predicted reflectance for an $\mathrm{Al}+\mathrm{AlF}_{3}$ reflector that would have an $\mathrm{AlF}_{3}$ thickness close to $3 \mathrm{~nm}$. This thickness estimate is based on work performed by Roodenko et,al through modelling the dielectric function of a $\mathrm{Al} / \mathrm{AlF}_{3} /$ Air system, where the $\mathrm{AlF}_{3}$ layer resulted from etching a $\mathrm{Si}$ sacrificial layer and passivation of the aluminum surface in a $\mathrm{XeF}_{2}$ reactor. ${ }^{8}$

\section{CONCLUSIONS}

In conclusion, this paper studied the feasibility of using the LAPPS process developed at NRL of using a lowtemperature electron plasma beam to etch away the native oxide layer from bare $\mathrm{Al}$ coatings and passivate the surface with a thin $\mathrm{AlF}_{3}$ layer. The LAPPS process was also used in a demonstration to reduce the thickness of the dielectric overcoat of $\mathrm{Al}$ test samples protected with $\mathrm{LiF}$ and $\mathrm{AlF}_{3}$. Measurements of the FUV reflectance before and after treatment showed a very significant reduction in reflectance for the sample coated with $\mathrm{LiF}$. These results suggest the LiF material may not be compatible with the e-beam etching treatment as this sample also exhibited a significant tarnishing of its surface. The second and third samples $\left(\mathrm{Al}+\mathrm{ALF}_{3}\right.$ and bare $\mathrm{Al}$ respectively) also showed a significant although smaller reduction in the FUV average reflectance (in comparison to the LiF sample). Future surface analysis will be performed on these samples to determine any changes that may have happened in terms of the chemical composition (at the atomic level) on the top-most layers. A separate etching experiment performed with $\mathrm{XeF}_{2}$ gas on a second bare $\mathrm{Al}$ with a native oxide layer yielded a slight increase in the average FUV reflectance that went from 39\% (pre-exposure) to $43 \%$ (post-exposure). Although it was not expected that the exposure to $\mathrm{XeF}_{2}$ gas alone would have removed the native oxide layer from this sample, the slight increase in FUV reflectance provides some encouragements to the future experiments that are planned in the research chamber (shown in Fig. 1) where fluorination of fresh $\mathrm{Al}$ samples will be performed by exposing them to $\mathrm{XeF}_{2}$ gas before oxidation would occur. A successful demonstration of these fluorination experiments on oxide-free $\mathrm{Al}$ samples would produce a thin $\mathrm{AlF}_{3}$ layer with an estimated thickness close to $3 \mathrm{~nm}^{8}$ The predicted reflectance performance of an $\mathrm{Al}$ sample coated uniformly with a $3 \mathrm{~nm}$ layer of $\mathrm{AlF}_{3}$ is shown on the right panel of Fig. 6 (green square symbols). These results indicate that such sample would yield a reflectance value of $50 \%$ at $100 \mathrm{~nm}$ and over $90 \%$ for wavelengths longer than $110 \mathrm{~nm}$. Finally, the environmental stability 
of an actual $\mathrm{Al}$ sample coated with an $\mathrm{AlF}_{3}$ protection layer is shown on the left side of Fig. 6. These results show a sample with an average FUV reflectance close to $74 \%$. Furthermore, this same sample does not exhibit any significant changes in terms of its FUV reflectance over a six months period after the sample was kept inside a regular storage box with a relative humidity close to $40 \%$.

\section{ACKNOWLEDGMENTS}

This work has been performed in the Optics Branch (Code 551) at the Goddard Space Flight Center (GSFC) and supported through a NASA Astrophysical Research and Analysis (APRA) grant No. 15-APRA15-0103 and a GSFC Internal Research and Development Program (IRAD) award. The authors wish to thank James Chervenak and Jose Santos (NASA-GSFC/553) for their support in the $\mathrm{XeF}_{2}$ etching done on one of the bare Al sample.

\section{REFERENCES}

[1] M.R. Bolcar, L. Feinberg, K. France, B.J. Rauscher, D. Redding, D. Schiminovich, "Initial technology assessment for the large-aperture uv-optical-infrared (luvoir) mission concept study," in [Space Telescopes and Instrumentation 2016], Loew, M. H., ed., Proc. SPIE 9904 (2016).

[2] E. Spiller, "Reflective multilayer coatings for the far-UV region," Appl. Opt. 15, 2333 (1976).

[3] R.A.M. Keski-Kuha, J.F. Osantowski, G.M. Blumenstock, J.S. Gum, C.M. Fleetwood, D.B. Leviton, T.T. Saha, J.G. Hagopian, J.L. Tveekrem, and G.A. Wright, "High reflectance coatings and materials for the extreme ultraviolet," Proc. SPIE 2428, 294-306 (1995).

[4] J. Tumlinson et al., "Unique Astrophysics in the Lyman Ultraviolet," ArXiv e-prints (2012).

[5] W.R. Hunter, J.F. Osantowski, and G. Hass, "Reflectance of aluminum overcoated with $\mathrm{MgF}_{2}$ and $\mathrm{LiF}$ in the wavelength region from $1600 \AA$ to $300 \AA$ at various angles of incidence," Appl. Opt. 10, 540 (1971).

[6] M.A. Quijada, S. Rice, and E. Mentzell, "Enhanced $\mathrm{MgF}_{2}$ and LiF Over-coated Al Mirrors for FUV Space Astronomy," Proc. SPIE 8450 (2012).

[7] M. A. Quijada, J. Del Hoyo, and S. Rice, "Enhanced far-ultraviolet reflectance of $\mathrm{MgF}_{2}$ and LiF over-coated Al mirrors," 9144, 91444G (2014).

[8] K. Roodenko, O. Seitz, Y. Gogte, J.-F. Veyan, X.-M. Yan, and Y. J. Chabal, "Modification of the Adhesive Properties of $\mathrm{XeF}_{2}$-Etched Aluminum Surfaces by Deposition of Organic Self-Assembled Monolayers," $J$. Phys. Chem. C 114, 2256622572 (2010).

[9] K. Roodenko, M. D. Halls, Y. Gogte, O. Seitz, J.-F. Veyan, and Y. J. Chabal, "Nature of Hydrophilic Aluminum Fluoride and Oxyaluminum Fluoride Surfaces Resulting from XeF2 Treatment of $\mathrm{Al}$ and $\mathrm{Al}_{3}$," J. Phys. Chem. C 115, 2135121357 (2011).

[10] S. G. Walton, D. R. Boris, S. C. Hernandez, E. H. Lock, Tz. B. Petrova, G. M. Petrov, and R. F. Fernsler, "Electron Beam Generated Plasmas for Ultra Low $\mathrm{T}_{e}$ Processing," Journal of Solid State Science and Technology 4, N5033-N5040 (2015).

[11] T.F. Zhang, J.Y. Park, W. Huang, G.A. Somorjai, "Influence of reaction with $\mathrm{XeF}_{2}$ on surface adhesion of $\mathrm{Al}$ and $\mathrm{Al}_{2} \mathrm{O}_{3}$ surfaces," Appl. Phys. Lett. 93, 141905 (2008).

[12] W. M. Manheimer, R. F. Fernsler, M. Lampe, R. A. Meger, "Theoretical overview of the large-area plasma processing system (LAPPS)," Plasma Sources Sci. Technol. 9, 370 (2000).

[13] R. A. Meger, R. F. Fernsler, M. Lampe, W. Manheimer, "large area plasma processing system," U.S. Patent $5,874,807(1999)$.

[14] G.M. Petrov, D.R. Boris, E.H. Lock, T.B. Petrova, R.F. Fernsler, S.G. Walton, "The influence of magnetic field on electron beam generated plasmas," J. Phys. D. Appl. Phys. 48, 275202-8 (2015).

[15] D. R. Boris, Tz. B. Petrova, G. M. Petrov, and S. G. Walton Journal of Vacuum Science 83 Technology A 35, 01A104 (2017).

[16] S. Wilbrandt, O. Stenzel,1,* H. Nakamura, D. Wulff-Molder, A. Duparr, N. Kaiser, "Protected and enhanced aluminum mirrors for the VUV," Applied Optics 53, No. 4 (2014). 